\title{
Privacy-aware electronic society
}

\author{
Maryline Laurent • Sara Foresti • Heng Xu
}

Published online: 8 November 2013

(C) Institut Mines-Télécom and Springer-Verlag France 2013

Our electronic society is making fast progress for offering users greater comfort in their daily activities. Users can benefit from fast access to services; better reactivity of their physical, computing, and networking environments to their own needs and habits; and preselection of information flows and relationships in which they are interested. Our digital society is eager to collect and analyze information for serving users as best as possible. Profiling users is a usual marketing activity, but new lucrative applications exploiting the huge amount of available information are emerging. The trend of Big Data has been accompanied by concerns regarding the aggregation, dissemination, and re-identification of personal identifiable information.

This special issue contributes to address these privacy issues, proposing privacy-enhancing technologies and solutions suited to the today's electronic society. The majority of the selected papers in this issue propose techniques and/or models for supporting privacy preferences and privacy-aware access control. The special issue also includes a contribution illustrating how the web browsing history can uniquely identify users for profiling purpose. Finally, there is a usercentered study discussing how much privacy people are willing to lose in order to gain some utilities. Collectively, these papers consider diverse contexts, ranging from social networks and web browsing to medical/social environments and Internet of Things (IoT).

\footnotetext{
M. Laurent $(\bowtie)$

Télécom SudParis, Évry, France

e-mail: maryline.laurent@telecom-sudparis.eu

S. Foresti

Università degli Studi di Milano, Milan, Italy

H. Xu

Pennsylvania State University, Pennsylvania, PA, USA
}

Said Oulmakhzoune, Nora Cuppens-Boulahia, Frédéric Cuppens, Stéphane Morucci, Mahmoud Barhamgi, and Djamal Benslimane propose a lightweight vocabulary for the fine-grained definition of privacy preferences in the paper titled "Privacy Query Rewriting Algorithm Instrumented by a Privacy-Aware Access Control Model." Data owners express their privacy requirements through the PrivOrBAC privacy-aware model, in terms of restrictions on consent, accuracy, purpose, and recipients. SPARQL queries submitted by users are then rewritten in such a way that the query results comply with data owners' privacy preferences. This rewriting process exploits PrivOrBAC privacy-aware model by decomposing SPARQL queries into a set of PrivOrBAC web services representing privacy preferences that must be satisfied. An interesting use case is also illustrated, showing the applicability of the approach in a healthcare scenario.

The paper "PriMa: A Comprehensive Approach to Privacy Protection in Social Network Sites" by Anna Squicciarini, Federica Paci, and Smitha Sundareswaran, proposes a privacy protection mechanism for social network (SN) users named "Privacy Manager" (PriMa). PriMa helps users, through dynamically generated access rules, to preserve the privacy of their traits, that is, their pieces of information exposed to other $\mathrm{SN}$ users. The rules are generated based on three criteria as follows: (1) the level of accessibility of the user's traits, (2) the profile of the owner's privacy preference for the specific trait or for the trait's category, and (3) the risks of unwanted or uncontrolled leakage of traits resulting from connected users who are eligible to access the profile owner's traits. PriMa is implemented and the performance evaluation demonstrates that access rule generation takes reasonable time less than $0.7 \mathrm{~ms}$ for 150 traits and 260 users.

The paper "Improving the User Content Privacy on Social Networks Using Rights Management Systems," by Joaquim Marques and Carlos Serrao, aims at protecting users' privacy in SNs. To overcome privacy issues arising when users share 
content through a $\mathrm{SN}$, the SN privacy and security sharing control system is complemented with a right management system, enforcing additional security and privacy mechanisms. These additional mechanisms protect users' privacy against the SN platform itself, besides from SN users who should not see some shared content. The adaptation of a digital right management system to the $\mathrm{SN}$ environment represents an interesting paradigm shift for controlling access to shared content, since such a control is moved from the remote service to the user side.

The paper titled "A Survey on Addressing Privacy Together With Quality of Context (QoC) for Context Management in the Internet of Things" by Sophie Chabridon, Romain Laborde, Thierry Desprats, Arnaud Oglaza, Pierrick Marie, and Samer Machara Marquez analyzes the privacy issues that arise when IoT offers context-aware services. Indeed, context-aware services represent a great opportunity for IoT, but they also pose new privacy concerns that limit their wide applicability. The authors describe the main criteria used to assess QoC, as well as privacy definitions and technologies, separately. The focus of the work is on the issues arising when combining QoC and privacy in the IoT scenario. In this scenario, the authors identify and discuss three main challenges as follows: (1) the decoupling of the production and consumption of context data, (2) the need to enable dynamic QoC-aware privacy policies, and (3) the complex interdependency of QoC and privacy.

The paper titled "On the Uniqueness of Web Browsing History Patterns," by Lukasz Olejnik, Claude Castelluccia, and Artur Janc, discusses how the history of browsing can uniquely identify most of the people who access the Internet. Furthermore, the authors show that users can be tracked based on their browsing histories, since the browsing histories rarely change and modifications are limited. Hence, browsing histories represent fingerprints of web users and pose new privacy threats that need to be mitigated. The results presented by the authors come from a wide experimental analysis of real browsing histories, which is illustrated in the paper. Privacy risks caused by the identifying nature of web browsing histories, as well as countermeasures to limit these breaches, are illustrated and discussed.

The paper titled "Privacy Concerns in Assisted Living Technologies," by Vaibhav Garg, L. Jean Camp, Lesa Lorenzen-Huber, Kalpana Shankar, and Kay Connelly, focuses on the intersection of an understudied group (nontechnical older adults) and sensitive medical data in a home environment. This work aims to examine privacy concerns among the aging population that requires the adoption of in-home and medical technologies to complement the traditional caregiver model. A four-dimensional framework that explains the tradeoffs between functionality and privacy among older adults was applied and examined as follows: (1) perceived utility, (2) data granularity, (3) data recipient, and (4) activity sensitivity. A survey study with 101 respondents was conducted to empirically examine the applicability and robustness of this framework.

Our grateful thanks go to the reviewers of the papers for this special issue. They carefully reviewed submissions made to this special issue and helped us selecting high-quality papers with solid scientific contributions. We also sincerely thank all contributing authors for considering submitting their works to this special issue and for their continuous efforts to improve their submissions until final approval. Last but not least, we thank the administrative staff of Annals of Telecommunications for their kind and helpful support. 